\title{
Cost-Minimization Analysis of Computed Tomography versus Magnetic Resonance Imaging in the Evaluation of Patients with Transient Ischemic Attacks at a Large Academic Center
}

\author{
Evgeny V. Sidorov ${ }^{a}$ Wuwei Feng $^{b}$ Magdy Selim ${ }^{c, d}$ \\ a Department of Neurology, Oklahoma University Health Sciences Center, Oklahoma City, \\ Okla., b Medical University of South Carolina, Charleston, S.C., ' Stroke Division, Department \\ of Neurology, Beth Israel Deaconess Medical Center, and 'Harvard Medical School, \\ Boston, Mass., USA
}

\author{
Key Words \\ Transient ischemic attack · Stroke · Cost · Evaluation · Magnetic resonance imaging · \\ Computed tomography
}

\section{Abstract}

Background: The type of neuroimaging for the evaluation of transient ischemic attack (TIA) is debatable. Many patients undergo head computed tomography (CT) with or without CT angiogram (CTA) while being at the emergency department (ED) and later magnetic resonance imaging (MRI) with MR angiogram (MRA) during admission. We hypothesized that evaluation with only one imaging modality (CT/CTA or MRI/MRA) is sufficient to formulate a treatment plan. We looked for the most cost-effective way to evaluate TIA patients. Methods: We performed a retrospective chart review of 82 patients with TIA. All patients had completely resolved neurological deficit at the time of their evaluation in the ED. We divided the patients into two groups. In group 1 , the evaluation included CT with CTA of the head and neck. In group 2, the evaluation included brain MRI with MRA or CTA of the head and neck. We compared these two groups for clinical characteristics and etiological evaluations of stroke/ TIA. The outcomes were measured by the number of therapeutic adjustments for the prevention of another ischemic stroke/TIA at the time of discharge from the hospital and revascularization procedures. We counted the following as therapeutic adjustment: (1) antiplatelet

The study was performed at Beth Israel Deaconess Medical Center, Harvard Medical School, Boston, Mass., USA. 
Sidorov et al.: Cost-Minimization Analysis of CT versus MRI in the Evaluation of Patients with TIA at a Large Academic Center

therapy was started de novo; (2) anticoagulation was started; (3) arterial revascularization procedure was performed, and (4) one antiplatelet agent was substituted for another. We performed a cost-effectiveness analysis if the outcomes of the two groups were different and a cost-minimization analysis if there was no difference in the outcomes. All cost calculations were made based on Medicare CPT codes. Results: Group 1 included 23 patients and group 259 patients. The patients in both groups had similar demographic and clinical characteristics. There was no difference in other etiological evaluations in groups 1 and 2. All patients underwent head CT as the first tool of evaluation whether MRI was done later or not. Therapeutic adjustments and revascularization procedures did not differ between the two groups. All head CTs showed no acute changes. MRI showed small ischemic infarcts in $44 \%$ of the patients in group 2. The average per-patient cost of neuroimaging with CT/CTA was USD 1,460.00, with CT and MRI/MRA USD 1,569 and with CT/CTA and brain MRI USD 2,090.00 ( $p<0.01)$. Conclusion: Either MRI/MRA or CT/CTA might be sufficient for the evaluation of patients with TIA or small asymptomatic strokes. If head CT at the ED is bypassed, a brain MRI with MRA of the head and neck would be the most informative tool at the lowest cost. Prospective studies with larger numbers of patients are needed for a better understanding of the safety and cost of imaging tools used for patients with TIA.

(C) 2014 S. Karger AG, Basel

\section{Introduction}

Patients with transient ischemic attack (TIA) often present to the emergency department (ED) when their symptoms have already improved [1]. A neurological examination is usually performed at baseline when patients are first evaluated by a physician. Head computed tomography (CT) is readily available from the ED and is frequently the first-choice test performed. Head CT is sensitive to exclude hemorrhage and shows clinically relevant ischemic infarcts in $4-13 \%$ of the patients with TIA [2,3]. Nonvascular pathology (tumor, abscess or subdural hematoma) was identified on CT scans in $1-5 \%$ of the TIA patients in various series $[4,5]$. Overall, CT provides limited information in most TIA patients. Including CT angiogram (CTA) of the head and neck into the evaluation helps diagnose vascular pathologies such as intracranial or extracranial stenosis $[6,7]$. It can also easily be done at the ED but requires iodinated contrast and may therefore not be performed on subjects with renal problems or who have an allergy to contrast agents [8]. Magnetic resonance imaging (MRI) is the most sensitive tool for ischemia evaluation and considered by some to be the preferred method of evaluation for TIA patients [9]. It shows positive changes on diffusion-weighted MR images in 35-81\% of the stroke patients initially diagnosed with TIA [10-14]. Like CTA, MR angiogram (MRA) of the head and neck provides information about vasculature pathology [7]. However, MRI/MRA often requires inpatient admission or much longer waiting time for evaluation given its limited availability.

Therefore, it is becoming increasingly common practice that CT and MRI studies are often combined and sometimes duplicated in stroke/TIA diagnosis workup. At the ED, TIA patients undergo head CT or even CTA, and later, on admission, their workup is extended with MRI/ MRA. This combined approach in the evaluation of patients with TIA is redundant because both imaging modalities provide similar information. Furthermore, it exposes patients to unnecessary radiation with $\mathrm{CT}$ and incurs increased health-care cost.

We hypothesized that evaluation with only one imaging modality (CT/CTA or MRI/MRA) is sufficient to formulate a treatment plan for patients with TIA. We performed this analysis to assess the clinical and cost-effectiveness (minimization) of CT- versus MRI-based neuroimaging evaluations in patients with TIA. 


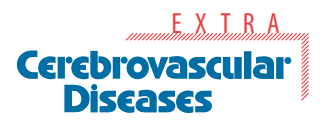

Table 1. CPT codes for the evaluation of TIA patients and Medicare reimbursement

\begin{tabular}{l|l}
\hline Cerebrovasc Dis Extra 2014;4:69-76 \\
\hline DOI: 10.1159/000360521 & $\begin{array}{l}\text { @ 2014 S. Karger AG, Basel } \\
\text { www.karger.com/cee }\end{array}$ \\
\hline
\end{tabular}

Sidorov et al.: Cost-Minimization Analysis of CT versus MRI in the Evaluation of Patients with TIA at a Large Academic Center

\begin{tabular}{llc}
\hline Name of the test & CPT codes & Cost, USD \\
\hline Head CT without contrast & 70450 & 237 \\
CTA head, including head CT & 70496 & 724 \\
CTA neck & 70948 & 736 \\
CT perfusion & $0042 \mathrm{~T}$ & 0 \\
MRI of the brain without contrast & 70551 & 630 \\
MRA brain & 70551 & 630 \\
MRA neck with contrast & 70548 & 702 \\
\hline
\end{tabular}

\section{Methods}

We conducted a retrospective review of consecutive patients with TIA and small strokes but completely resolved neurological deficit who presented to the ED of the Beth Israel Deaconess Medical Center as 'code stroke' during a 2-year period from 2009 to 2010.

\section{Data Collection Procedure}

The following information was retrieved from clinical charts: demographic data (age, gender, time from the onset of symptoms to ED presentation and symptoms duration); clinical characteristics (presence of diabetes mellitus, hypertension, hyperlipidemia, atrial fibrillation and congestive heart failure with an ejection fraction $<30 \%$ ); tests performed for an etiological evaluation of stroke/TIA (transthoracic and transesophageal echocardiogram, hemoglobin $\mathrm{A} 1 \mathrm{C}$ and lipid panel); medications at presentation to the ED, new medications started upon initial evaluation at the ED or by hospital discharge; and length of hospitalization. We also retrieved the official interpretation of the imaging studies by the neuroradiologists.

\section{Study Groups}

The patients were divided into two groups according to the neuroimaging evaluations. The evaluation in group 1 included head CT and head and neck CTA and in group 2 a brain MRI and either a head and neck CTA or MRA.

\section{Outcome Measures}

To assess the impact of imaging modalities on therapeutic decision-making, we determined the number of patients who had therapeutic adjustments made to their admission medications at the time of discharge based on MRI/MRA results. We considered the following as treatment adjustments: (1) antiplatelet therapy was started de novo; (2) anticoagulation was started; (3) arterial revascularization procedure was performed, and (4) one antiplatelet agent was substituted for another. We planned to perform a cost-effectiveness analysis if therapeutic adjustments or arterial revascularizations were different between the groups and a cost-minimization analysis if they were similar, based on CT and MRI CPT coding for Medicare in Boston, Mass., USA (table 1).

\section{Statistical Analysis}

Analyses were performed using Microsoft Excel for windows. Categorical variables (gender, presence of diabetes mellitus, hypertension, hyperlipidemia, atrial fibrillation and congestive heart failure) were statistically analyzed using $\chi^{2}$ analysis, and continuous variables (age, blood pressure and cost of imaging tool) were analyzed using Student's test. Statistical significance was set at $\mathrm{p}<0.05$. 
Sidorov et al.: Cost-Minimization Analysis of CT versus MRI in the Evaluation of Patients with TIA at a Large Academic Center

Table 2. Baseline demographic and clinical characteristics of the patients

\begin{tabular}{lccc}
\hline & $\begin{array}{c}\text { Group 1 } \\
(\mathrm{n}=23)\end{array}$ & $\begin{array}{l}\text { Group 2 } \\
\text { (n=59) }\end{array}$ & p value \\
\hline Male & $15(65)$ & $30(50)$ & 0.24 \\
Age, years & $67 \pm 17$ & $69 \pm 14$ & 0.68 \\
Time from symptom onset to ED, min & $145 \pm 143$ & $186 \pm 224$ & 0.42 \\
TIA symptom duration, min & $34 \pm 40$ & $42 \pm 43$ & 0.47 \\
Length of hospitalization, days & $2.13 \pm 1.39$ & $2.03 \pm 0.82$ & 0.82 \\
Diabetes mellitus & $4(17)$ & $17(28)$ & 0.32 \\
Hypertension & $17(74)$ & $44(75)$ & 0.50 \\
Hyperlipidemia & $12(52)$ & $39(66)$ & 0.24 \\
Atrial fibrillation & $6(26)$ & $7(12)$ & 0.06 \\
Left ventricular ejection fraction <30\% & $2(9)$ & $3(5)$ & 0.54 \\
Antiplatelet use prior to the event & $11(48)$ & $27(46)$ & 0.86 \\
Anticoagulant use prior to the event & $7(30)$ & $10(17)$ & 0.17 \\
Systolic blood pressure at ED, mm Hg & $146 \pm 20$ & $149 \pm 25$ & 0.60 \\
Diastolic blood pressure at ED, mm Hg & $79 \pm 11$ & $75 \pm 14$ & 0.24 \\
\hline
\end{tabular}

Values represent $\mathrm{n}(\%)$ or mean $\pm \mathrm{SD}$.

Table 3. Therapeutic adjustments and endarterectomy of the patients in group 1 and group 2

\begin{tabular}{llcc}
\hline Treatment change at the time of hospital discharge & Group 1 (n=23) & Group 2 (n=59) & p value \\
\hline No change & $8(35)$ & $10(17)$ & 0.08 \\
Antiplatelet started & $5(22)$ & $21(36)$ & 0.23 \\
Antiplatelet changed & $5(22)$ & $21(36)$ & 0.23 \\
Anticoagulation started (ED + inpatient) & $5(22)$ & $6(10)$ & 0.17 \\
Anticoagulation started (inpatient) & $1(4)$ & $3(5)$ & 0.88 \\
Carotid endarterectomy & $0(0)$ & $2(3)$ & 0.37 \\
\hline
\end{tabular}

Values represent $\mathrm{n}(\%)$.

\section{Results}

\section{Study Groups}

A total of 82 patients ( 45 men and 37 women) were included in the study. Baseline demographic and clinical characteristics are presented in table 2. All patients underwent head CT scanning at presentation at the ED as part of the hospital 'code stroke' protocol. Most of the patients came to the ED within 2-3 h of symptom onset. MRI was performed in 59 patients (group 2), and 23 patients were only evaluated with head CT (group 1). There was no difference in baseline demographic or clinical characteristics in patients who were or were not evaluated with MRI. There was also no difference in the length of stay or other etiological evaluations such as echocardiogram or lipid panel.

\section{Therapeutic Adjustments and Arterial Revascularization for TIA Patients at Discharge}

Patients were either started on an antiplatelet therapy (mostly aspirin) or anticoagulation de novo, or their antiplatelet therapy was changed from one agent to another. The results are presented in table 3. The total number of adjustments adds up to 83 because 1 
patient had carotid endarterectomy and was switched from aspirin to clopidogrel. Arterial revascularization procedures were performed in $3.4 \%$ of the patients in group 2 versus $0 \%$ in group 1 ( $\mathrm{p}=0.27$ ). Other therapeutic adjustments did not differ between the patients who were evaluated with MRI and those who were not $(p>0.05)$ (table 3$)$.

\section{Neuroimaging Findings}

Head CT done at the ED upon presentation did not show acute changes (early signs of ischemia or brain hemorrhage) in any of the 82 patients. Out of the 59 patients who were evaluated with diffusion-weighted MRI, 26 patients (44\%) had acute small infarcts without changes on the neurological examination. Three of these 26 patients $(11.5 \%)$ were started on anticoagulation after MRI had been done. One case had a stump embolus, another was discovered to have a hypercoagulable state and the third case had paroxysmal atrial fibrillation. Two patients (7.7\%) had their aspirin changed to clopidogrel after the MRI scan. The therapeutic changes in all of these 5 patients were independent of their MRI findings. In patients whose MRI was normal, there were no therapeutic adjustments during inpatient stay. In group 1, only 1 patient with adjustment of antiplatelet at the ED was started on anticoagulation during inpatient stay because of atrial fibrillation on cardiac telemetry.

\section{Cost-Minimization Analysis}

The majority of the patients in this study had CT/CTA at the ED and later MRI for further evaluation. The average per-patient cost of neuroimaging with CT/CTA was USD 1,460.00, with CT and MRI/MRA USD 1,569 and with CT/CTA and brain MRI USD 2,090.00 ( $<<0.01)$.

\section{Discussion}

Similar to other previous reports, we found that imaging TIA patients with MRI provides more information, such as the detection of acute small infarcts with changes in the diffusionweighted imaging sequence, than with CT. However, we found no difference in therapeutic adjustments made between TIA patients who underwent CT/CTA and those who underwent MRI/MRA. Performing MRI/MRA on patients who already had CT/CTA added to the overall cost without therapeutic value. Our results indicate that the evaluation of TIA patients with only one imaging modality (CT/CTA or MRI/MRA) minimizes the cost and is sufficient to formulate a treatment plan.

We only performed a cost-minimization analysis but no cost-effectiveness analysis, as we found no difference in therapeutic adjustments based on CT- versus MRI-based imaging. Imaging with CT/CTA was the least costly. In general, all combinations of neuroimaging studies are acceptable for TIA patients, but repeating one study after another, such as evaluating a patient with brain MRI after head CT, is unnecessary.

Patients with TIA may be admitted as inpatients for evaluation or managed as outpatients in dedicated TIA clinics, depending on the hospital and the country $[11,12]$. In our institution, patients with TIA are admitted for observation and expedited workup. Both CTand MRI-based imaging modalities are commonly used in the evaluation of patients with TIA. This is consistent with the current American Heart Association (AHA)/American Stroke Association (ASA) TIA evaluation guidelines $[1,15,16]$. The current AHA Get With the Guidelines (GWTG) and Primary Stroke Center (PSC) quality requirements recommend that patients presenting within $3 \mathrm{~h}$ of stroke symptom onset undergo brain imaging within 25 min of arrival at the ED [17]. This prompted us to limit this study to TIA patients for whom 'code stroke' was activated. Our institutional policy is to activate 'code stroke' for patients arriving within $9 \mathrm{~h}$ of symptom onset. We found that despite complete resolution of neurological 
Sidorov et al.: Cost-Minimization Analysis of CT versus MRI in the Evaluation of Patients with TIA at a Large Academic Center

symptoms, head CT was routinely performed at the ED [18]. This is in line with current practice in many hospitals, given the widespread availability and easy accessibility to CT (as opposed to MRI) and ability to meet the GWTG and PSC benchmarks [17]. In our cohort, all head CT scans were negative for acute findings. Other studies that evaluated the usefulness of head CT in patients with TIA reported that localization-related ischemia or hemorrhage can be found in a small number of patients [2, 3]. In TIA patients whose CT scan is unrevealing, workup is usually extended to include brain MRI to determine the exact cause. If MRI shows small ischemic infarcts, it helps to confirm the ischemic nature of patient symptoms. MRI may also suggest the source of embolism; multiple bilateral small infarcts may indicate a proximal source of embolization from the heart or the aortic arch. In this study, although a numerically higher proportion of patients had their therapeutic regimen changed during their inpatient stay after MRI had been performed, this was not significantly different between the two study groups [15]. In addition, the decision to start a new antithrombotic therapy in these patients was independent of their MRI results. This raises the questions of whether (1) initial head CT evaluation is necessary in patients with TIA and (2) TIA patients should undergo MRI even if CT has already been done. In our cohort, we found that additional imaging with MRI did not provide information that resulted in a change in therapy based on CT imaging, suggesting that CT/CTA may still be sufficient for TIA evaluation in most cases.

When considering the cost, we found that the imaging combination CT + CTA + MRI had the highest cost (USD 2,090). The cost of evaluation with CT + MRI + MRA (USD 1,569), on the other hand, was comparable to that of CT + CTA (USD 1,460). In this case, if initial head CT was not done, the cost would be USD 237 less. Thus, making the evaluation with MRI + MRA less costly than that with CT + CTA. This is because MRA of the brain is a time-of-flight modality of noncontrast brain MRI and does not require additional study. Thus, both evaluations are incorporated in one CPT code and billed only once. MRA of the neck often requires contrast administration and is therefore billed separately. Noncontrast head CT with CTA of the head, on the other hand, requires contrast and is more expensive than MRI. CTA of the neck also has a separate CPT code [19].

Our study is limited by its retrospective nature, its lack of systematic long-term follow-up data and a relatively small number of patients in a single center. We only included patients who presented to the ED within $9 \mathrm{~h}$ of symptom onset. Therefore, our findings may not be generalizable to other centers or all TIA patients. However, the observed trend in TIA evaluation where CT is almost always performed before MRI is commonly seen at several other hospitals in the United States. This study only accounts for the direct cost of neuroimaging, but not the cost of hospitalization or other diagnostic tests and treatments. Although this could lead to some uncertainties regarding the equivalence of the cost of additional tests between the study groups, it is unlikely that further tests such as telemetry and echocardiogram are influenced by the imaging results. In fact, we found no difference in the number of other diagnostic tests performed between the two groups. Lastly, we found no difference in the length of hospitalization between the two study groups. This likely reflects the easy access to and ability to perform MRI 24/7 at the ED of our institution. It is likely that the length of hospitalization, and therefore the overall cost could increase at other institutions where access to MRI is more limited.

\section{Conclusion}

TIA patients should ideally be evaluated directly with MRI/MRA, if available in a timely manner. This is both more informative and cost-effective. Initial evaluation with head CT in these patients is unnecessary. If brain MRI is unavailable, evaluation with CT/CTA is still suffi- 
cient to formulate a treatment plan. Repeating MRI following CT/CTA may reveal small infarcts but is unlikely to change the treatment plan and adds more to the overall cost. Future prospective multicenter studies with long-term follow-up data in a larger cohort of TIA patients are needed to better understand the safety and cost of imaging for TIA.

\section{Disclosure Statement}

There are no conflicts of interest to declare.

\section{References}

1 Easton JD, Saver JL, Albers GW, et al: Definition and evaluation of transient ischemic attack: a scientific statement for healthcare professionals from the American Heart Association/American Stroke Association Stroke Council; Council on Cardiovascular Surgery and Anesthesia; Council on Cardiovascular Radiology and Intervention; Council on Cardiovascular Nursing; and the Interdisciplinary Council on Peripheral Vascular Disease. The American Academy of Neurology affirms the value of this statement as an educational tool for neurologists. Stroke 2009;40:2276-2293.

-2 Koudstaal PJ, van Gijn J, Frenken CW, Hijdra A, Lodder J, Vermeulen M, Bulens C, Franke CL: TIA, RIND, minor stroke: a continuum, or different subgroups? Dutch TIA Study Group. J Neurol Neurosurg Psychiatry 1992;55: 95-97.

3 Douglas VC, Johnston CM, Elkins J, Sidney S, Gress DR, Johnston SC: Head computed tomography findings predict short-term stroke risk after transient ischemic attack. Stroke 2003;34:2894-2898.

4 Weisberg LA: Computerized tomographic abnormalities in patients with hemispheric transient ischemic attacks. South Med J 1986;79:804-807.

-5 Turnbull IW, Bannister CM: CT observations on the natural history of asymptomatic cerebral infarction following transient ischaemic attacks. Neurol Res 1985;7:190-193.

6 Coutts SB, Modi J, Patel SK, Demchuk AM, Goyal M, Hill MD: CT/CT angiography and MRI findings predict recurrent stroke after transient ischemic attack and minor stroke: results of the prospective CATCH study. Stroke 2012;43:1013-1017.

7 Purroy F, Jimenez Caballero PE, Gorospe A, et al: Prediction of early stroke recurrence in transient ischemic attack patients from the PROMAPA study: a comparison of prognostic risk scores. Cerebrovasc Dis 2012;33: 182-189.

-8 Smith WS, Roberts HC, Chuang NA, Ong KC, Lee TJ, Johnston SC, Dillon WP: Safety and feasibility of a CT protocol for acute stroke: combined CT, CT angiography, and CT perfusion imaging in 53 consecutive patients. AJNR Am J Neuroradiol 2003;24:688-690.

-9 Furie KL, Kasner SE, Adams RJ, Albers GW, Bush RL, Fagan SC, Halperin JL, Johnston SC, Katzan I, Kernan WN, Mitchell PH, Ovbiagele B, Palesch YY, Sacco RL, Schwamm LH, Wassertheil-Smoller S, Turan TN, Wentworth D: Guidelines for the prevention of stroke in patients with stroke or transient ischemic attack: a guideline for healthcare professionals from the American Heart Association/American Stroke Association. Stroke 2011;42: 227-276.

10 Ovbiagele B, Kidwell CS, Saver JL: Epidemiological impact in the United States of a tissue-based definition of transient ischemic attack. Stroke 2003;34:919-924.

11 Olivot JM, Albers GW: Diffusion-perfusion MRI for triaging transient ischemic attack and acute cerebrovascular syndromes. Curr Opin Neurol 2011;24:44-49.

-12 Fazekas F, Fazekas G, Schmidt R, Kapeller P, Offenbacher H: Magnetic resonance imaging correlates of transient cerebral ischemic attacks. Stroke 1996;27:607-611.

13 Bhadelia RA, Anderson M, Polak JF, Manolio TA, Beauchamp N, Knepper L, O'Leary DH: Prevalence and associations of MRI-demonstrated brain infarcts in elderly subjects with a history of transient ischemic attack. The Cardiovascular Health Study. Stroke 1999;30:383-388.

14 Chatzikonstantinou A, Wolf ME, Schaefer A, Hennerici MG: Risk prediction of subsequent early stroke in patients with transient ischemic attacks. Cerebrovasc Dis 2013;36:106-109.

15 Amort M, Fluri F, Weisskopf F, Gensicke H, Bonati LH, Lyrer PA, Engelter ST: Etiological classifications of transient ischemic attacks: subtype classification by TOAST, CCS and ASCO - a pilot study. Cerebrovasc Dis 2012; 33:508-516.

-16 Amort M, Fluri F, Schafer J, Weisskopf F, Katan M, Burow A, Bucher HC, Bonati LH, Lyrer PA, Engelter ST: Transient ischemic attack versus transient ischemic attack mimics: frequency, clinical characteristics and outcome. Cerebrovasc Dis 2011;32:57-64. 
17 Alberts MJ, Latchaw RE, Jagoda A, Wechsler LR, Crocco T, George MG, Connolly ES, Mancini B, Prudhomme S, Gress D, Jensen ME, Bass R, Ruff R, Foell K, Armonda RA, Emr M, Warren M, Baranski J, Walker MD: Revised and updated recommendations for the establishment of primary stroke centers: a summary statement from the brain attack coalition. Stroke 2011;42:2651-2665.

18 Diaz-Guzman J, Egido JA, Gabriel-Sanchez R, Barbera-Comes G, Fuentes-Gimeno B, Fernandez-Perez C: Stroke and transient ischemic attack incidence rate in Spain: the IBERICTUS study. Cerebrovasc Dis 2012;34:272281.

19 http://www.ama-assn.org/ama/pub/physician-resources/solutions-managing-your-practice/codingbilling-insurance/cpt.page? 2012. 